\title{
Normal Venous Phase Documented during Angiography in Patients with Spinal Vascular Malformations: Incidence and Clinical Implications
}

\author{
(DD. Eckart Sorte, DM. Obrzut, (D)E. Wyse, and DP. Gailloud
}

\begin{abstract}
BACKGROUND AND PURPOSE: A key angiographic sign observed in patients with spinal vascular malformations is the absence of a normal venous phase. While this finding alone is often believed to rule out a lesion impacting the perimedullary venous drainage, the observation of a venous phase in several patients with vascular malformations led us to reconsider the validity of that sign.
\end{abstract}

MATERIALS AND METHODS: Eighty-one patients with 6 spinal arteriovenous malformations, 16 perimedullary arteriovenous fistulas, 61 spinal epidural or dural AVFs, and 1 paravertebral AVF (2 patients had multiple lesions) were reviewed. The venous phase was defined as normal, absent, or indeterminate. The venous phase timing was analyzed in patients with spinal dural or epidural AVFs.

RESULTS: The existence of a venous phase could not be determined for technical reasons in 23 patients. A venous phase was documented in 25 of 58 patients (43\%), including 16 of 49 vascular malformations (40.0\%) with perimedullary venous drainage. Twelve of the 30 patients $(40.0 \%)$ with dural or epidural AVFs had a normal venous phase, appearing, on average, 10.1 seconds and best visualized 15.0 seconds after opacification of the artery of Adamkiewicz.

CONCLUSIONS: A normal venous phase was observed in $43 \%$ of patients with spinal vascular malformations, and within an acceptable delay ( $<18$ seconds) in $40 \%$ of slow-flow AVFs. While it remains an important angiographic sign, the observation of a normal venous phase cannot be used to exclude the presence of a vascular malformation or justify interrupting a diagnostic spinal angiogram.

ABBREVIATIONS: SAVM = spinal AVM; SDAVF = spinal dural arteriovenous fistula; SEAVF = spinal epidural arteriovenous fistula; SpDSA = spinal digital subtraction angiography; $S V M=$ spinal vascular malformation

S pinal digital subtraction angiography (SpDSA) is the criterion standard imaging technique for the evaluation of the spinal vasculature and remains essential for the diagnosis and management of spinal vascular malformations (SVMs). ${ }^{1}$ The practice of SpDSA requires a sound understanding of the vascular anatomy of the spinal cord, notably its venous system. ${ }^{2,3}$ A key angiographic sign observed in patients with SVMs is the absence of a normal venous phase, a phenomenon first reported in 2 cases of spinal dural arteriovenous fistulas (SDAVFs). ${ }^{2}$ It was later suggested that a spinal angiogram could be terminated when "a nor-

Received June 4, 2015; accepted after revision July 22.

From the Division of Interventional Neuroradiology, The Johns Hopkins Hospital, Baltimore, Maryland.

Paper previously presented in part at: American Society of Neuroradiology Annual Meeting and the Foundation of the ASNR Symposium, May 17-22, 2014; Montreal, Quebec, Canada.

Please address correspondence to: Philippe Gailloud, MD, Division of Interventional Neuroradiology, The Johns Hopkins Hospital, Bloomberg 7218, $1800 \mathrm{E}$ Orleans St, Baltimore, MD 21287; e-mail: phg@jhmi.edu

http://dx.doi.org/10.3174/ajnr.A4601 mal venous phase is visualized and the veins correspond to the defects on the myelogram," an approach based on the assumption that "if the venous phase of the spinal circulation is normal, this alone rules out DAVF [dural AVF] as the cause of the patient's symptoms." 4 While MR imaging has now supplanted myelography in the work-up of spinal vascular anomalies, the notion that the angiographic pursuit of an SVM can stop after the documentation of a normal venous phase is still widely accepted, though at times more cautiously. Some authors have, for example, suggested that a normal venous phase "usually" or "reportedly" 6 allows terminating a diagnostic angiogram or that it only makes the "diagnosis of a fistula less likely." 7 To our knowledge, there is, so far, only 1 reported instance of a normal venous phase associated with a vascular malformation draining into the perimedullary system, but this case involved a cranial dural arteriovenous fistula rather than an SVM. ${ }^{8}$

Several observations of a morphologically normal venous phase associated with lesions involving the perimedullary venous system have pushed us to reconsider the validity of this angiographic sign. The spinal venous phase was, therefore, 

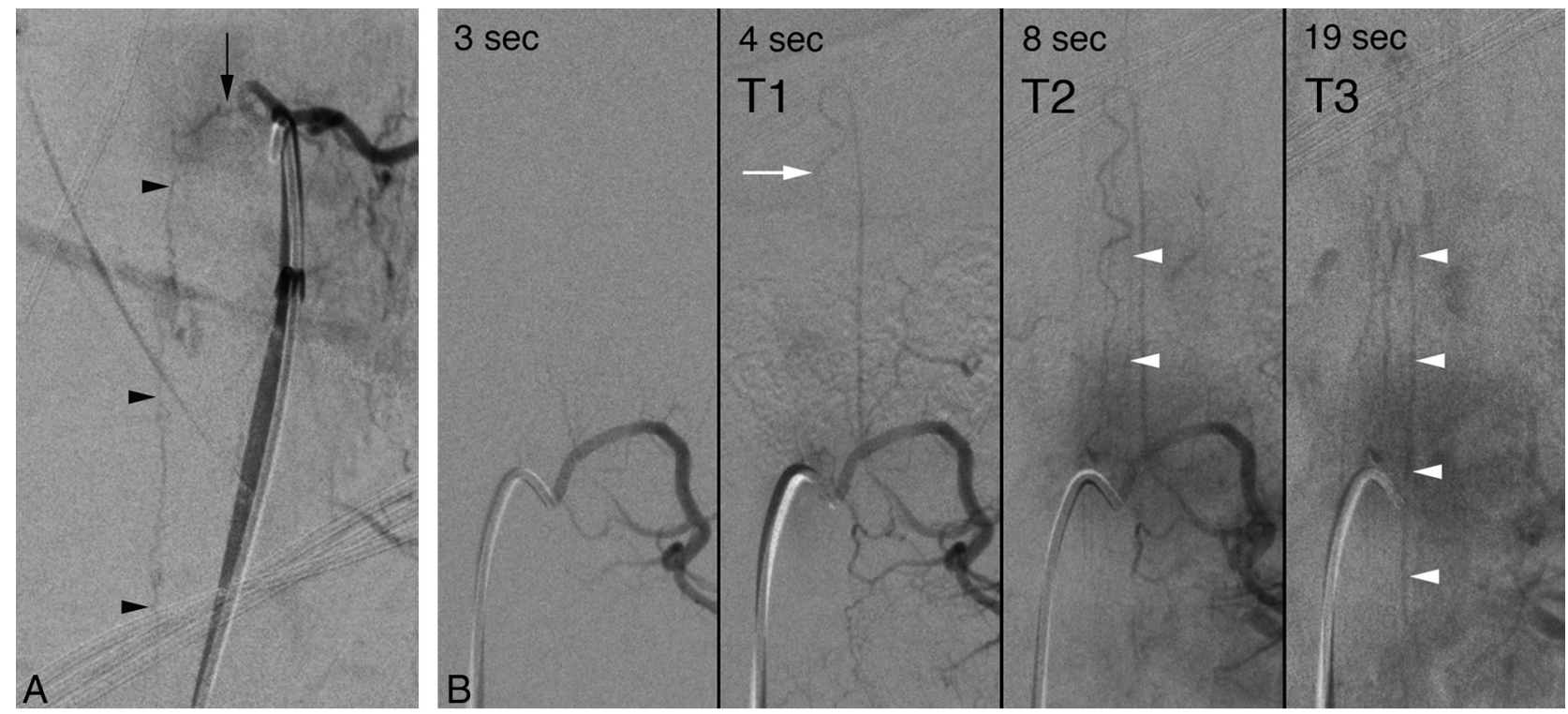

FIG 1. Timing of the spinal venous phase in a 51-year-old man with a progressive myelopathy secondary to a left T8 spinal dural arteriovenous fistula. A, SpDSA, left T8 injection, posteroanterior view, documents an SDAVF (black arrow) with perimedullary venous drainage (black arrowheads). B, SpDSA, left L1 injection, posteroanterior view, demonstrates the opacification sequence of the medullary circulation and the time stamps used to calculate the spinal venous phase: $T 1$ indicates initial opacification of the anterior spinal artery (white arrow) (4 seconds); T2, initial detection of perimedullary structures, including the proximal segment of the left L2 radiculomedullary vein (white arrowheads) (8 seconds); and T3, best visualization of the spinal venous phase (19 seconds). In this case, the time to initial detection and best delineation was 4 and 15 seconds, respectively.

Spinal venous phase in slow-flow arteriovenous fistulas with perimedullary venous drainage

\begin{tabular}{lccc}
\multicolumn{1}{c}{ Feature } & Overall & Venous Phase Absent & Venous Phase Present \\
\hline No. of lesions & $30(100 \%)$ & $18(60.0 \%)$ & $12(40.0 \%)$ \\
SDAVF & 11 & $8(44.4 \%)$ & $3(25 \%)$ \\
SEAVF & 17 & $9(50.0 \%)$ & $8(66.7 \%)$ \\
SDAVF/SEAVF & 2 & $1(5.6 \%)$ & $1(8.3 \%)$ \\
Male predominance & $26 / 30(86.7 \%)$ & $14 / 18(77.8 \%)$ & $12 / 12(100 \%)$ \\
Age (average) & 65 & 66.6 & 64.7 \\
Symptomatic & $29 / 30(96.7 \%)$ & $18 / 18(100 \%)$ & $11 / 12(91.2 \%)$ \\
Flow voids on MRI & $19(63.3 \%)$ & $10 / 18(55.6 \%)$ & $9 / 12(75.0 \%)$ \\
Thoracic location & 11 & $6(33.3 \%)$ & $5(41.7 \%)$ \\
Lumbar location & 11 & $8(44.4 . \%)$ & $3(25.0 \%)$ \\
Sacral location & 8 & $4(22.2 \%)$ & $4(33.3 \%)$ \\
\hline
\end{tabular}

The angiographic venous phase was evaluated after opacification of the artery of Adamkiewicz and was classified as normal, absent, or indeterminate. The venous phase timing was analyzed in the subgroup of patients with slow-flow arteriovenous fistulas (SEAVF/SDAVF). We recorded 3 time stamps: initial opacification of the anterior spinal artery ( $\mathrm{t} 1)$, initial detection of perimedullary venous structures ( $\mathrm{t} 2$ ), and best delineation of the spinal venous phase $(\mathrm{t} 3)$. The times evaluated in 81 patients diagnosed with an SVM in our practice during a 5-year period, with particular attention paid to SDAVFs and spinal epidural arteriovenous fistulas (SEAVFs).

\section{MATERIALS AND METHODS}

This study is based on 81 patients logged in an institutional review board-approved data base between February 2010 and February 2015, including 23 females (28\%) and 58 males (72\%), with ages ranging from 19 months to 89 years (average, 49 years; median, 23 years). Only lesions with arteriovenous shunting were considered. Seventy-nine patients had a single lesion, while 2 patients had 2 and 3 lesions respectively, for a total of 84 SVMs. The lesions included 39 SEAVFs, 20 SDAVFs, 1 paraspinal arteriovenous fistula, 6 spinal arteriovenous malformations (SAVMs), and 16 perimedullary arteriovenous fistulas. In 2 instances, a slow-flow fistula could not be clearly classified as SEAVF or SDAVF. The multiple lesions were all SEAVFs. at which the venous phase was first $(\mathrm{t} 2-\mathrm{t} 1)$ and best documented $(\mathrm{t} 3-\mathrm{t} 1)$ were then calculated (Fig 1$)$.

\section{RESULTS}

The existence of a venous phase could not be determined for technical reasons (eg, short acquisitions, motion artifacts) in 23 patients $(28.4 \%)$. A venous phase was seen in 25 of the remaining 58 patients (43\%), including all 9 cases of SVM without perimedullary venous drainage, principally consisting of paraspinal arteriovenous fistulas or high-flow SEAVFs in children.

\section{Spinal Venous Phase in SVMs with Perimedullary Venous Drainage}

A normal venous phase was seen in 16 of the 49 patients $(32.7 \%)$ with angiographically documented perimedullary venous drainage, including 1 of 14 perimedullary arteriovenous fistulas $(7.1 \%), 3$ of 5 SAVMs (all in cervical or cervi- 

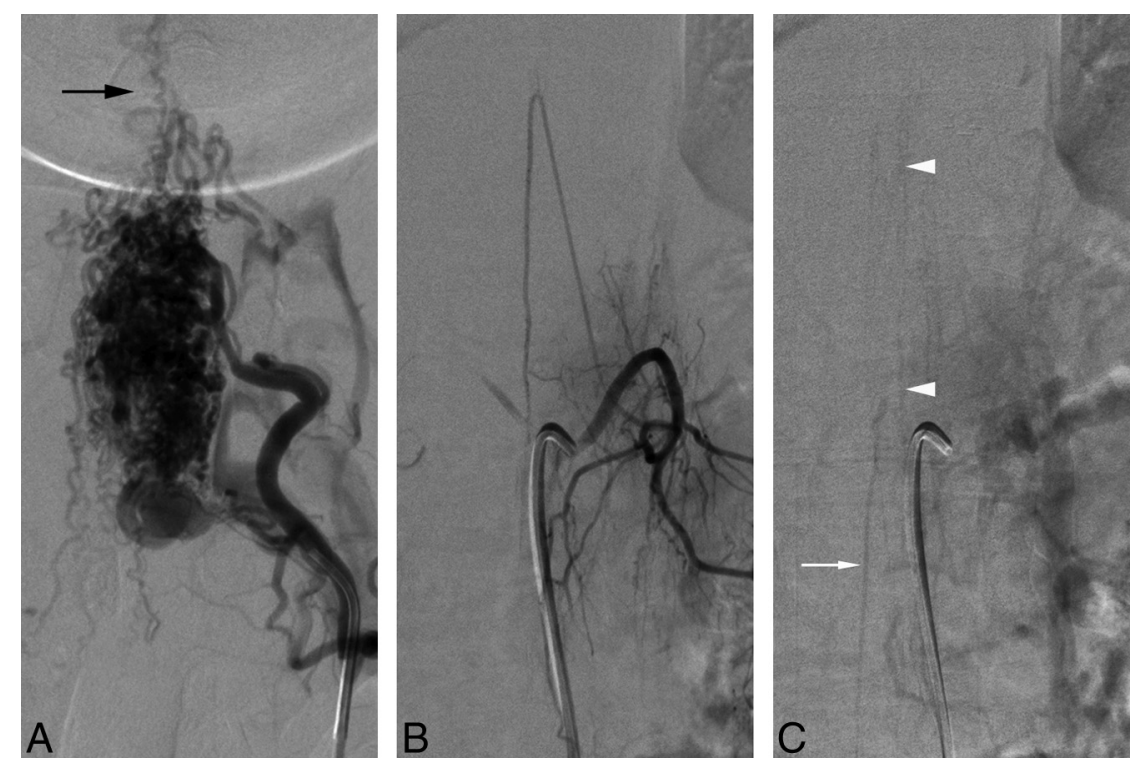

FIG 2. A 21-year-old man with a spinal arteriovenous malformation. A, DSA, left costocervical trunk injection, posteroanterior view, documents a large cervicothoracic SAVM nidus. Note that the venous drainage essentially occurs at the level of the lesion or in a cranial direction (black arrow). B, SpDSA, left T12 injection, posteroanterior view, arterial phase with opacification of the artery of Adamkiewicz. C, SpDSA, left T12 injection, posteroanterior view, later phase, documents the normal perimedullary venous drainage, including the visualization of spinal (white arrowheads) and radiculomedullary (white arrow) veins.

cothoracic locations) (60.0\%), and 12 of 30 SEAVFs/SDAVFs $(40.0 \%)$.

\section{Spinal Venous Phase in Slow-Flow Arteriovenous Fistulas with Perimedullary Venous Drainage}

The 30 patients with SEAVFs/SDAVFs draining into the perimedullary venous system are summarized in the Table. They included 4 women and 26 men, with ages ranging between 49 and 89 years (average, 65 years; median, 65 years). The lesions were located between T3 and S1; all were symptomatic except 1 SDAVF incidentally noted during preoperative tumor embolization. A normal venous phase was detected in 12 of these patients (40\%). In particular, a lumbosacral radiculomedullary draining vein was documented in 9 of the 10 cases that included the lumbosacral region in the FOV. The type and topographic distribution of the fistulas were comparable between the groups with and without a venous phase. While our series is too limited for definitive statistical analysis, it appears that lesions located in the lumbar region lacked a venous phase $(72.7 \%)$ more often than the thoracic or sacral fistulas (54.5\% and 50\%, respectively) and that SDAVFs lacked a venous phase more often than SEAVFs (72.7\% versus $52.9 \%)$. In all cases, the vertebral levels at which the abnormal perimedullary drainage and the normal venous phase were observed overlapped.

The average time for the beginning of the venous phase was 10.1 seconds (range, $4-29$ seconds; median, 8 seconds), and the average time for the best delineation of the venous phase was 15.0 seconds (range, 7-39 seconds; median, 15 seconds). If 18 seconds is used as the upper limit for the observation of a normal venous phase, ${ }^{6}$ a delayed venous phase was observed in 1 instance (29 seconds). When this outlying patient with respective values of 29 and 39 seconds was omitted (case illustra- tion 5), the average times for the beginning and best delineation of the venous phase were 8.4 seconds (range, 4-14 seconds; median, 7.5 seconds) and 12.8 seconds (range, 7-18 seconds; median, 14.5 seconds), respectively.

\section{Case Illustrations}

Besides the patient demonstrating the method used to time the venous phase (Fig 1), 6 illustrative cases have been selected, including 1 SAVM, 2 SEAVFs, and 3 SDAVFs.

Case 1. A 21-year-old man with a 5-month history of progressive lower extremity weakness was diagnosed with a cervical SAVM after he had a posterior fossa hemorrhage. He was referred to our service 3 months later for further evaluation. SpDSA confirmed the diagnosis of SAVM supplied by the right vertebral artery and both costocervical trunks (Fig 2A). The injection of the artery of Adamkiewicz, found at the left T12, showed a normal venous phase (Fig $2 B,-C$ ).

Case 2. A 64-year-old man presented with a 9-month history of leg weakness associated with urinary retention and constipation, progressively worsening until he became wheelchairbound. MR imaging revealed an intramedullary T2 signal abnormality without perimedullary flow voids. SpDSA documented a right S1 SEAVF with extensive perimedullary venous drainage (Fig $3 A$ ). The injection of the artery of Adamkiewicz, found at the left T11, showed a normal venous phase (Fig 3B,-C).

Case 3. A 71-year-old man with an 8-month history of progressive lower extremity weakness, initially diagnosed as transverse myelitis, presented with a sudden deterioration of his bowel and bladder function before becoming wheelchair-bound. MR imaging showed intramedullary T2 signal abnormalities without perimedullary flow voids. SpDSA revealed a right S1 SEAVF with perimedullary drainage (Fig $4 A,-B$ ). The injection of the artery of Adamkiewicz, found at the left T8, showed a normal venous phase (Fig 4C, -D).

Case 4. A 58-year-old man was referred for preoperative embolization of a spinal metastasis from renal cell carcinoma. SpDSA incidentally documented an SDAVF supplied by the right L1 intersegmental artery (Fig 5A). The injection of the artery of Adamkiewicz, found at left L1, showed a normal venous phase (Fig $5 B,-C$ ).

Case 5. A 66-year-old man had a progressive right lower extremity weakness associated with paresthesias and pain. MR imaging showed intramedullary T2 signal abnormalities without perimedullary flow voids. A work-up for infectious and inflammatory etiologies remained negative. SpDSA revealed a 

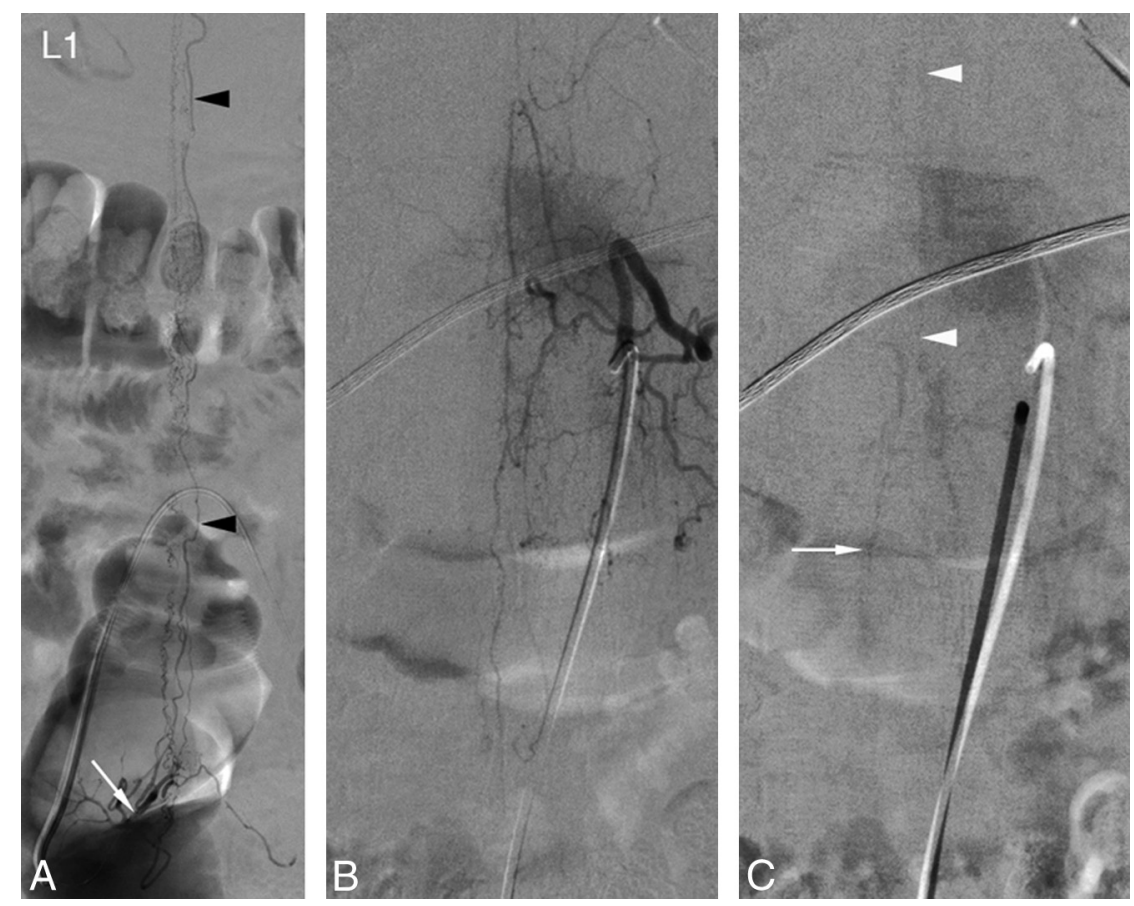

FIG 3. A 64-year-old man with a right $\mathrm{S} 1$ spinal epidural arteriovenous fistula. $A, S p D S A$, right S1 injection, posteroanterior view. The selective injection of the right SI SEAVF (white arrow) reveals extensive perimedullary drainage (black arrowheads) from the sacral region up to about T5 (above L1 is not shown in this image). B, SpDSA, left T11 injection, posteroanterior view, arterial phase with opacification of the artery of Adamkiewicz. C, SpDSA, left T11 injection, posteroanterior view, later phase, documents the normal perimedullary venous drainage, including the visualization of spinal (white arrowheads) and radiculomedullary (white arrow) veins. Venous phase timing: first detection $=14$ seconds, best delineation $=18$ seconds. right L1 SDAVF (Fig 6A). The injection of the artery of Adamkiewicz, found at the left T12, documented a normal venous phase (Fig 6B).

Case 6. A 65-year-old man with a recent history of heart transplantation and duodenal neuroendocrine tumor presented with rapidly worsening back pain, quadriparesis, and paresthesias. MR imaging showed perimedullary flow voids without definite intramedullary signal abnormality. SpDSA demonstrated a left T3 SDAVF (Fig 7A). The injection of the artery of Adamkiewicz, found at left $\mathrm{T} 9$, revealed a normal venous phase (Fig $7 B,-C)$.

\section{DISCUSSION}

\section{Classification and Diagnosis of Spinal Vascular Malformations}

Spinal vascular anomalies with arteriovenous shunts can pragmatically be divided into 2 main categories. The high-flow lesions include the SAVMs, the fast perimedullary arteriovenous fistulas (Merland types 2 and 3), ${ }^{9}$ and some high-output SEAVFs, generally of congenital or traumatic nature. The slow-flow lesions include SDAVFs,
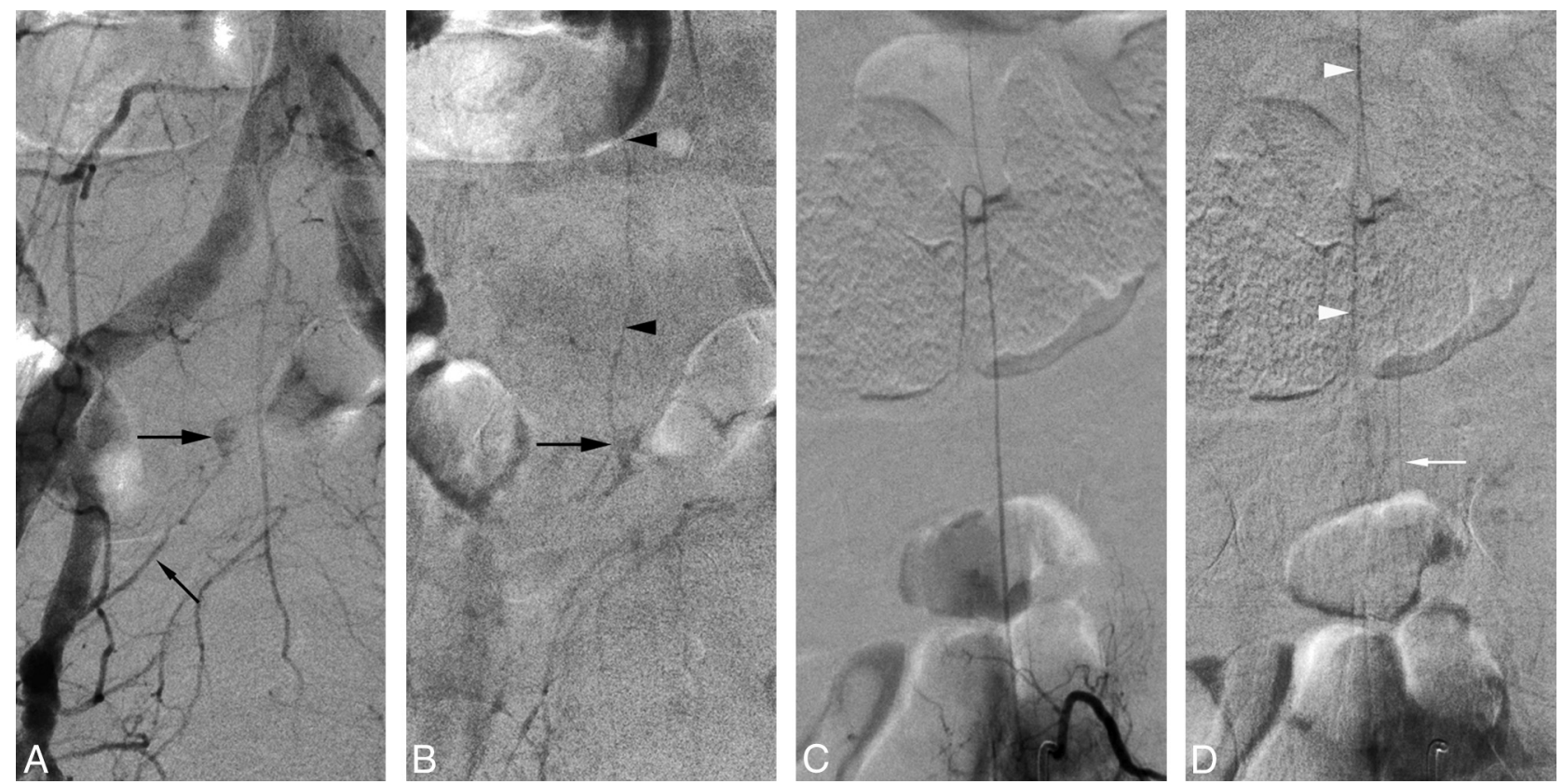

FIG 4. A 71-year-old patient with a right S1 spinal epidural arteriovenous fistula. A, SpDSA, right S1 injection, posteroanterior view; the selective injection of the right S1 radicular artery (small black arrow) opacifies the epidural venous pouch of the SEAVF (large black arrow). B, SpDSA right S1 injection, posteroanterior view; a slightly later phase shows the drainage of the isolated epidural pouch (large black arrow) into a right S1 radiculomedullary vein (black arrowheads). C, SpDSA, left T11 injection, posteroanterior view, arterial phase with opacification of the artery of Adamkiewicz. D, SpDSA, left T11 injection, posteroanterior view, later phase, documenting the normal perimedullary venous drainage, including the visualization of spinal (white arrowheads) and radiculomedullary (white arrow) veins. Venous phase timing: first detection $=7$ seconds, best delineation $=13$ seconds. 

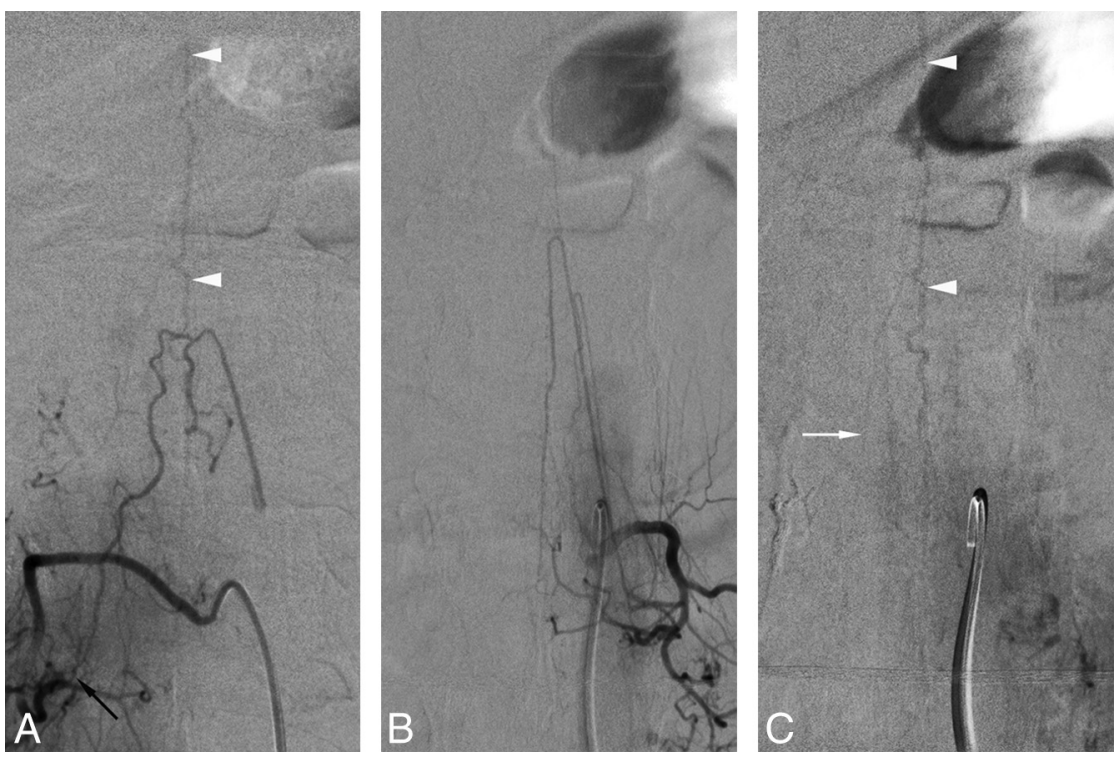

FIG 5. A 58-year-old man with a right $\mathrm{L} 1$ spinal dural arteriovenous fistula. A, SpDSA, right L1 injection, posteroanterior view, documents a right LI SDAVF (black arrow) draining into the perimedullary venous system (white arrowheads). B, SpDSA, left L1 injection, posteroanterior view, arterial phase with opacification of prominent anterior and posterior radiculomedullary arteries (artery of Lazorthes). C, SpDSA, left L1 injection, posteroanterior view, later phase, documents the normal perimedullary venous drainage, including the visualization of spinal (white arrowheads) and radiculomedullary (white arrow) veins. Note that the opacified longitudinal spinal axis is identical to the one seen draining the SDAVF in A. Venous phase timing: first detection $=7$ seconds, best delineation $=10$ seconds.
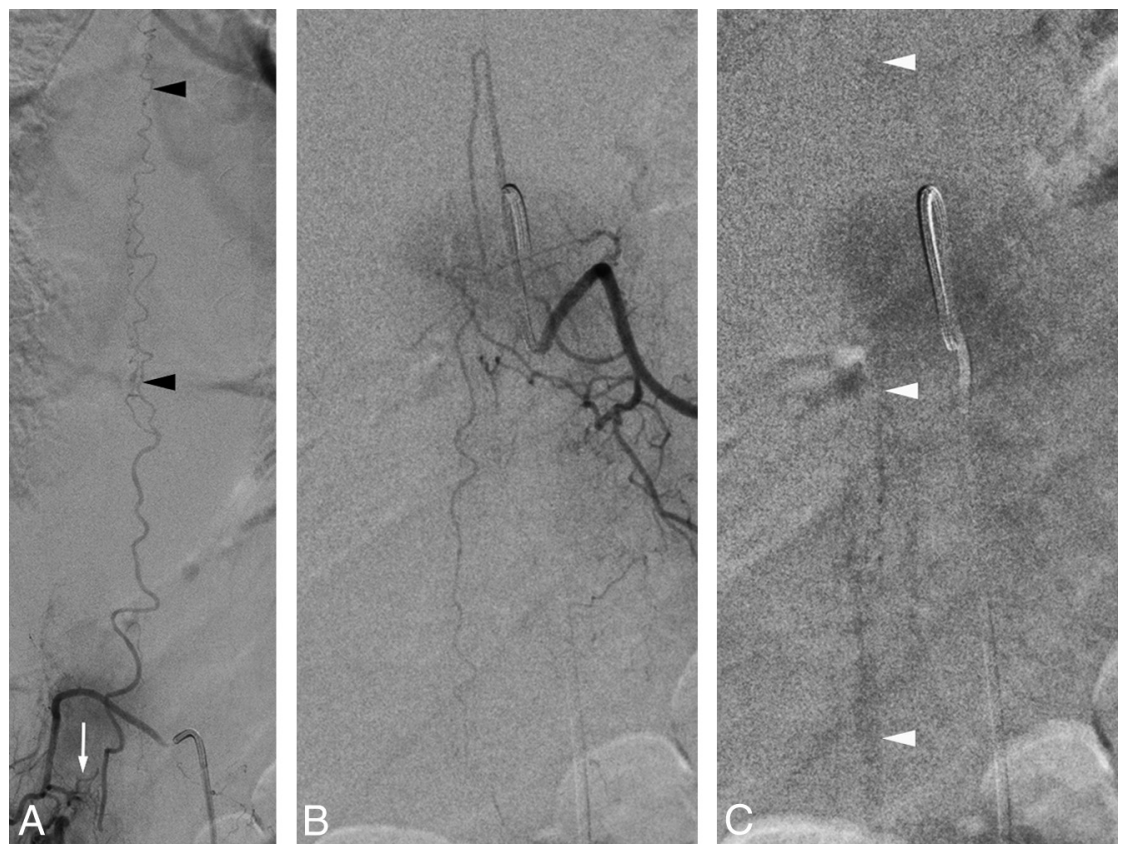

FIG 6. A 66-year-old man with a right L1 spinal dural arteriovenous fistula. A, SpDSA, right L1 injection, posteroanterior view, documents a right LI SDAVF (white arrow) draining into the perimedullary venous system (black arrowheads). B, SpDSA, left T12 injection, posteroanterior view, arterial phase with opacification of the artery of Adamkiewicz. C, SpDSA, left T12 injection, posteroanterior view, later phase, documents the normal perimedullary venous drainage, including the visualization of perimedullary veins (white arrowhead). A radiculomedullary vein is not clearly identified in this case. Venous phase timing: first detection $=29$ seconds, best delineation $=39$ seconds

SEAVFs, and the slow perimedullary arteriovenous fistula (Merland type 1).

From a diagnostic viewpoint, high-flow SVMs are usually easily identified by noninvasive imaging methods. On the other hand, the nonspecific MR imaging changes associated with slow-flow arteriovenous fistulas can be deceptive, particularly in the absence of typical flow voids. A recent study found that the sensitivity and positive predictive value of MR imaging for SVMs in general could be as low as $51 \%$ and $48 \%$, respectively. ${ }^{10}$ Our cohort similarly suggested a limited value for MR imaging, notably for slow-flow lesions, which were associated with an inaccurate initial diagnosis in $65 \%$ of cases. ${ }^{11}$ This low predictive value may be related to the importance generally attributed to the absence of detectable flow voids, which were only noted in 49 of our 81 patients $(60.5 \%)$. This situation is particularly relevant to our report because the angiographic documentation of a normal venous phase is generally used to rule out the presence of a slow-flow arteriovenous fistula in patients with inconclusive MR imaging findings.

\section{Spinal Angiography Technique}

The fundamental principles of selective spinal angiography established by its pioneers remain valid to this day. ${ }^{12-14}$ While partial studies may be performed in certain circumstances (eg, the limited diagnostic portion of a therapeutic procedure or follow-up angiograms), a typical diagnostic study includes at least the selective catheterization of each pair of intersegmental arteries and a pelvic flush aortogram (documenting an SVM does not rule out the presence of additional lesions ${ }^{1}$ ), often complemented by selective injections of the internal iliac, subclavian, vertebral, supreme intercostal, and (in some instances) carotid arteries. $^{4,6,15}$ The appropriate technique and equipment allow performing a complete spinal angiogram with the patient under conscious sedation with short procedure durations, reasonable radiation and contrast agent doses, and extremely low complication rates. ${ }^{10}$ However, a sign reliably permitting terminating the procedure early, such as the observation of a normal venous phase, would carry an important clinical value. The emphasis placed on reliability is particularly important because it has been es- 


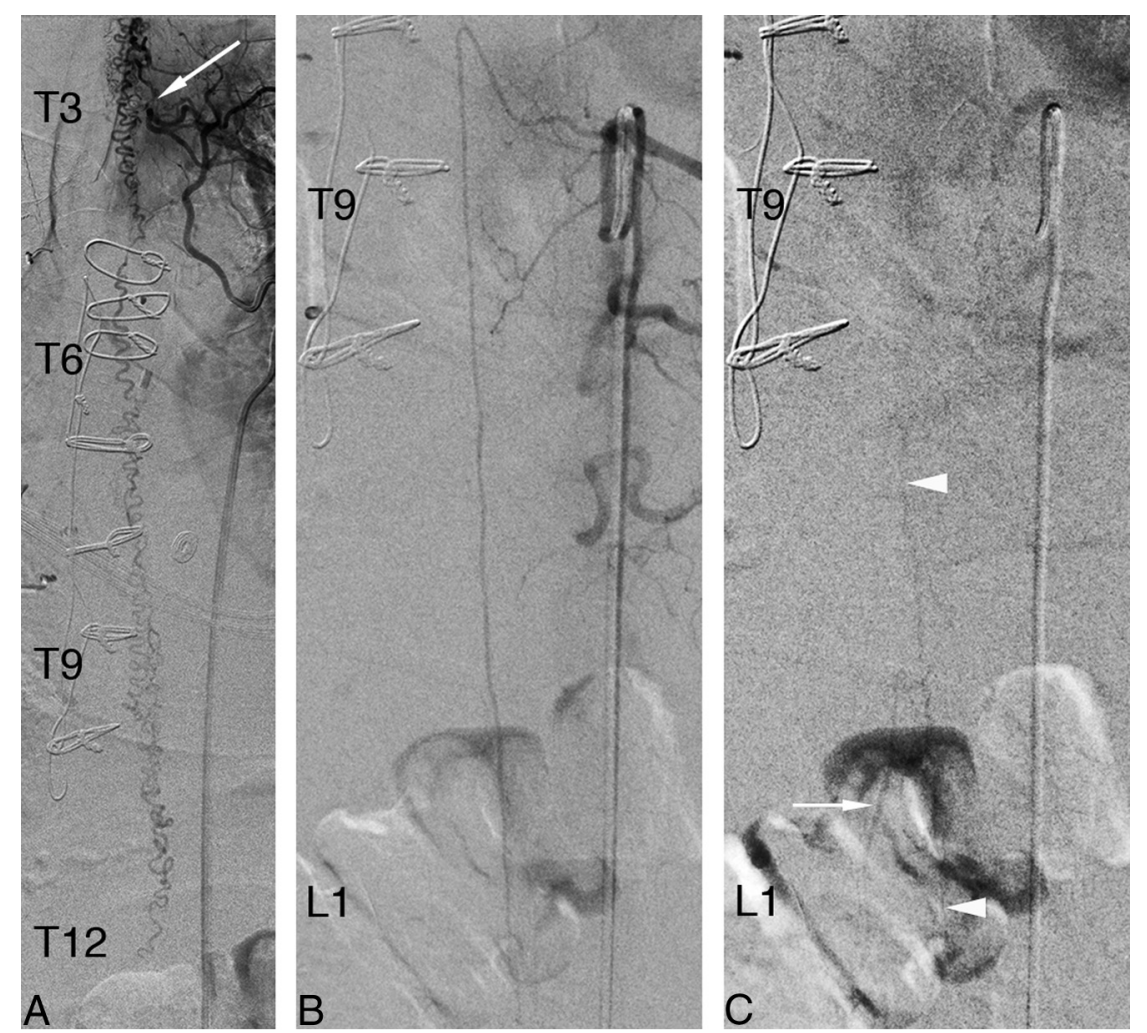

FIG 7. A 65-year-old man with a left T3 spinal dural arteriovenous fistula. A, SpDSA, left T3 injection, posteroanterior view, documents a left T3 SDAVF (white arrow) with extensive perimedullary venous drainage. $B$, SpDSA, left T9 injection, posteroanterior view, arterial phase with opacification of the artery of Adamkiewicz. C, SpDSA, left T9 injection, posteroanterior view, later phase, documents the normal perimedullary venous drainage, including the visualization of spinal (white arrowheads) and radiculomedullary (white arrow) veins. Venous phase timing: first detection $=11$ seconds, best delineation $=15$ seconds.

timated that about $50 \%$ of so-called false-negative findings on spinal angiograms result from a failure to investigate the vessel supplying the SVM. ${ }^{16}$

\section{Normal Venous Phase of Spinal Angiography}

Multiple factors influence the detectability of normal venous structures during the injection of the artery of Adamkiewicz, including the quality of the angiographic equipment, the patient's age and body habitus, the use of general anesthesia or conscious sedation (including the use of paralytic agents or the patient's ability to breath-hold when studied awake), and the presence of significant motion and/or bowel artifacts. The presence of a normal venous phase may, therefore, be underestimated by our retrospective study, in which a substantial portion of angiograms (28.4\%) were technically inadequate for the detection of small venous structures, principally because of respiratory and gastrointestinal motion artifacts or limited angiographic sequences in patients generally studied under conscious sedation. It is possible that performing diagnostic SpDSA with the patient under general anesthesia (by using paralytics and pausing the ventilator during injections) would reduce motion artifacts, particularly at the end of long angiographic sequences, and increase the visibility of small venous structures and the number of normal venous phases identified in patients with spinal vascular malformations. Yet, despite these drawbacks, $43 \%$ of all the reviewed SVMs were associated with a normal venous phase, including $40 \%$ of slow-flow lesions. The venous phase was analyzed at the lower thoracic and lumbosacral levels of the spinal cord (ie, the venous return of the anterior spinal artery after injection of the artery of Adamkiewicz). Some of the SVMs were, therefore, remotely located normal venous structures, such as a cervical cord SAVM (case 1) or an upper thoracic SDAVF (case 6), but most drained at the levels opacified during the observed normal venous phase. An interesting point made by Trop et al in $1998^{8}$ in regard to their cranial arteriovenous fistula with perimedullary drainage but associated with a normal venous phase was the normal MR imaging appearance of the lower spinal cord in their patient, which led them to conclude that "venous compartmentalization accounted for the isolated abnormal signal of the cervical cord and the normal venous drainage of the lower cord at MR imaging." This idea of a "longitudinal compartmentalization" could certainly apply to our 3 cases with remotely located SVMs.

Cases in which the normal and abnormal venous phases were documented at the same vertebral levels may suggest a relative functional independence of the anterior and posterior longitudinal venous systems, one preferentially participating in the drainage of the SVM while the other is involved in the visualization of a normal venous phase. Due to the 2D nature of SpDSA, however, the distinction between anterior and posterior spinal veins is generally not possible without the acquisition of lateral projections, which were generally not obtained in our patients. The existence of a "transverse compartmentalization" of the perimedullary venous drainage, therefore, remains hypothetic.

\section{The Mechanisms Underlying the Visualization or Nonvisualization of a Spinal Venous Phase in Perimedullary Venous Hypertension}

In our series, the lack of a spinal venous phase was slightly more common in SDAVFs than SEAVFs and in lesions located at the lumbar level. Both factors seem to favor a role for locoregional venous pressure variations. Hence, the observation of a venous phase, typically made at the level of the conus medullaris, would be more likely prevented by the pressure gradient produced by a closely rather than remotely located fistula (ie, lumbar versus sacral or thoracic). Similarly, the pressure gradient associated with an SDAVF, which consists of an arteriovenous shunt directly involving a radiculomedullary vein, should have a more significant locoregional impact than the gradient resulting from an 
SEAVF, in which the arteriovenous shunt is located on an epidural venous pouch with secondary drainage into a radiculomedullary vein (and other possible venous outflow pathways).

Case illustration 5 (Fig 6) suggests that the visualization of a normal venous phase may also depend on the existence of a patent radiculomedullary vein locally available to drain the perimedullary venous blood. In terms of venous timing, this case was a clear outlier (first documentation at 29 seconds, best delineation at 39 seconds) and could have easily been considered as lacking a normal venous phase without a long angiographic acquisition. Most interesting, this "transitional case" is also the one in which a patent lumbosacral radiculomedullary vein was not documented. It is, therefore, reasonable to think that a disappearing venous phase correlates with increasing venous drainage impairment and venous hypertension, a phenomenon typical of slow-flow arteriovenous fistulas well-described by Merland et al in 1980: ${ }^{17}$ "Hyperpressure is due to the presence of the shunt but also to impaired venous return, which in turn probably depends upon the absence of the veins normally draining into the epidural space."

\section{CONCLUSIONS}

A normal venous phase was observed in $43 \%$ of our patients, including all the SVMs without perimedullary drainage and all cervical or cervicothoracic junction SAVMs. More important, $40 \%$ of the slow-flow lesions (SEAVFs/SDAVFs) were associated with a normal venous phase as well. While the timing of the venous phase appeared to be delayed in that latter group, it was still within accepted normal limits (18 seconds) in all except 1 case. The principal implication of our report is that while it remains an interesting angiographic sign, the observation of a normal venous phase cannot be used to rule out the presence of an SVM or justify interrupting a diagnostic spinal angiogram.

Disclosures: Danielle Eckart Sorte-UNRELATED: Consultancy: Codman Neurovascular, Comments: nominal consulting fees for lectures; Payment for Lectures (including service on Speakers Bureaus): Codman Neurovascular (nominal consulting fees for lectures). Philippe Gailloud—UNRELATED: Consultancy: Codman Neurovascular; Grants/Grants Pending: Siemens*; Stock/Stock Options: ArtVentive Medical. *Money paid to the institution.

\section{REFERENCES}

1. Koch C. Spinal dural arteriovenous fistula. Curr Opin Neurol 2006; 19:69-75 CrossRef Medline

2. Launay M, Chiras J, Bories J. Angiography of the spinal cord: venous phase-normal features. pathological application [in French]. J Neuroradiol 1979;6:287-315 Medline

3. Tadié M, Hemet J, Freger P, et al. Morphological and functional anatomy of spinal cord veins [in French]. J Neuroradiol 1985;12:3-20 Medline

4. Willinsky R, Lasjaunias $\mathrm{P}$, Terbrugge $\mathrm{K}$, et al. Angiography in the investigation of spinal dural arteriovenous fistula: a protocol with application of the venous phase. Neuroradiology 1990;32:114-16 CrossRef Medline

5. Krings T, Geibprasert S, ter Brugge K. Case-Based Interventional Neuroradiology. New York: Thieme; 2011

6. Hurst RW. Spinal angiography. In: Baum S, ed. Abrams' Angiography Vascular and Interventional Radiology. Boston: Little, Brown and Company; 1997:356-88

7. Harrigan MR, Deveikis JP. Handbook of Cerebrovascular Disease and Neurointerventional Technique. Totowa, NJ: Humana Press; 2009

8. Trop I, Roy D, Raymond J, et al. Craniocervical dural fistula associated with cervical myelopathy: angiographic demonstration of normal venous drainage of the thoracolumbar cord does not rule out diagnosis. AJNR Am J Neuroradiol 1998;19:583-86 Medline

9. Gueguen B, Merland JJ, Riche MC, et al. Vascular malformations of the spinal cord: intrathecal perimedullary arteriovenous fistulas fed by medullary arteries. Neurology 1987;37:969-79 CrossRef Medline

10. Chen J, Gailloud P. Safety of spinal angiography: complication rate analysis in $\mathbf{3 0 2}$ diagnostic angiograms. Neurology 2011;77:1235-40 CrossRef Medline

11. Sorte D, Wyse E, Orru E, et al. Initial MRI diagnosis in 132 cases of angiographically confirmed spinal vascular malformations [SNIS oral abstract O-014]. J NeuroIntervent Surg 2015;7(suppl 1):A8 CrossRef

12. Di Chiro G, Doppman J, Ommaya AK. Selective arteriography of arteriovenous aneurysms of spinal cord. Radiology 1967;88:1065-77 CrossRef Medline

13. Djindjian R, Houdart R, Hurth M. Acquisitions récentes en angiographie médullaire. Revure Neurologique 1966;115:1068-69

14. Djindjian R, Hurth M, Houdart E. Angiography of the Spinal Cord. Baltimore: University Park Press; 1970

15. Gailloud P. Arterial anatomy of the spine and spinal cord. In: Mauro MA, Murphy KP, Thomson KR, et al, eds. Image-Guided Interventions. 2nd ed. Philadelphia: Saunders; 2014

16. Gailloud P, Wyse E, Greenberg B, et al. Analysis of 24 false negative spinal angiograms performed in 16 patients with vascular malformations subsequently documented by angiography: what went wrong and how to avoid it. In: Proceedings of the 52nd Annual Meeting of the American Society of Neuroradiology, Montréal, Quebec, Canada. May 17-22, 2014

17. Merland JJ, Riche MC, Chiras J. Intraspinal extramedullary arteriovenous fistulae draining into the medullary veins [in French]. J Neuroradiol 1980;7:271-320 Medline 